\title{
SITUACIÓN ACTUAL DE LA PROFILAXIS ANTIBIÓTICA EN EL REFLUJO VESICOURETERAL PRIMARIO EN EL NIÑO
}

\author{
Gloria Mำ Fraga Rodríguez, Joaquín Escribano Subias' y Esther Benito Acin.
}

Unidad de Nefrología Pediátrica. Hospital de la Santa Creu i Sant Pau. Universidad Autónoma de Barcelona. Barcelona.

Unidad de Nefrología Pediátrica'. Hospital Universitario Sant Joan. Universidad Rovira i Virgili. Reus. España.

\begin{abstract}
Resumen.- OBJETIVO: Valorar la eficacia de la profilaxis antibiótica en la prevención de las infecciones urinarias y del daño parenquimatoso renal en niños con Reflujo Vesicoureteral primario (RVU).
\end{abstract}

METODOS/RESULTADO. Se realiza una búsqueda de la literatura en MEDLINE y The Cochrane Library seleccionando ensayos clínicos y metaanálisis que comparen la profilaxis antibiótica (continua ó discontinua) y placebo ó ningún tratamiento en niños con RVU primario. Seleccionamos 3 revisiones sistemáticas que evaluan la eficacia de la profilaxis para prevenir las infecciones de orina y que incluyen estudios en los cuales predomina una población de niños sin RVU conocido. Los resultados mostraron que los antibióticos redujeron el riesgo de infección urinaria. La calidad de los estudios fue deficiente y por lo tanto los resultados son cuestionables.
Seleccionamos además dos ensayos clínicos en niños con reflujo, uno con información limitada pues se desconoce el grado de RVU y otro que evalua los resultados en una población de 113 niños con RVU grado I a III I55 con profilaxis y 58 sin profilaxis) después de una pielonefritis aguda. No existen diferencias en el riesgo de infección urinaria ni tampoco en el riesgo de lesión en el parénquima renal.

CONCLUSIONES. Las pruebas que apoyan el uso generalizado de antibióticos para prevenir las infecciones urinarias son débiles. No se ha demostrado un beneficio de la profilaxis en los RVU grado I a III. No se dispone de información en RVU de alto grado. Se requieren estudios adicionales para poder establecer unas recomendaciones más precisas con el objetivo de prevenir las infecciones de orina en niños con RVU.

Palabras clave: Reflujo vesicoureteral. Infección urinaria. Profilaxis antibiótica. Metanálisis.

Summary.- OBJECTIVES: To assess the efficacy of antibiotic prophylaxis for prevention of urinary infections and renal parenchymal damage in children with primary vesicoureteral reflux (VUR).

METHODS/RESULTS. A search based on MEDLINE and The Cochrane Library was performed selecting those cli-nical trials and meta-analysis which compared antibiotic prophylaxis leither continuous or intermittent) and place-bo or no treatment at all in children with primary VUR. Three systematic reviews were chosen for assessing the efficacy of prophylaxis of urinary infections including trials with a predominant paediatric population without known VUR. Results showed that the use of antibiotics decreased the risk of urinary infection. The quality of the trials was, however, insufficient and therefore of questionable results. We also selected two randomized controlled trials in children with reflux: one had limited information as the degree of reflux was not stated; the second assessed the 
results in a population of 173 children with VUR grade I to III (55 receiving prophylaxis and 58 not) fo-llowing acute pyelonephritis. There were no differences with regard to the risk of urinary infection or the risk of renal parenchymal damage.

CONCLUSIONS. There is not enough evidence supporting generalized use of antibiotics to prevent urinary infections. No benefit in prophylaxis has been proven for VUR grades I to III. There is no data for high grade VUR. It will be necessary to perform more trials in order to establish more accurate recommendations on prevention of urinary infections in the presence of VUR.

Keywords: Vesicoureteral reflux. Urinary tract infection. Antibacterial prophylaxis. Meta-analysis. Systematic review. Children.

\section{INTRODUCCIÓN}

El reflujo vesicoureteral (RVU) se define como el paso retrógrado de la orina a los uréteres y, frecuentemente, al sistema colector renal. La verdadera prevalencia en niños sanos es desconocida pero se estima entre un $1-3 \%$. En contraste, la prevalencia de RVU en niños con historia de una ó mas infecciones urinarias es de un $30-50 \%$.

Esta asociación lleva al concepto de que el RVU juega un papel en la patogénesis de las infecciones urinarias. La importancia de estas infecciones urinarias es su relación directa con la aparición de nuevas cicatrices renales que puedan comprometer la función renal futura, derivar en una hipertensión arterial y complicaciones en el embarazo.

La relación entre $\mathrm{RVU}$, infección del tracto urinario (ITU) y daño renal fue reportada tempranamente (1).

El daño renal permanente en niños después de una pielonefritis aguda (PNA) se ha estimado que ocurre en $5-20 \%$ de casos, valorado mediante urografía intravenosa. Con el uso de la gammagrafía renal, esta incidencia alcanza el $40 \%$ (2)(3). La incidencia de daño renal aumenta con cada episodio de PNA.

Tras la primera infección urinaria febril, el RVU se establece en el $39 \%$ de los niños menores de 2 años (4).

Por tanto la búsqueda de un reflujo en estos niños es esencial y está justificada si se admite que la profilaxis antibiótica permite reducir la recidiva de las infecciones urinarias y prevenir las lesiones renales parenquimatosas.

En la actualidad las opciones terapéuticas disponibles son el manejo médico que incluye, básicamente, la profilaxis antibiótica y medidas higiénico-dietéticas y el tratamiento mediante una técnica intervencionista (reimplante ureteral ó inyección endoscópica).

Los estudios que comparan antibióticos sólo versus antibióticos más cirugía no han mostrado un beneficio adicional de la cirugía salvo una reducción en el riesgo de ITU febril.

En este artículo nos centraremos en un aspecto del tratamiento médico del RVU que es la profilaxis antibiótica y la evidencia científica disponible que valore la eficacia de la misma para prevenir las infecciones urinarias y el daño renal. Para ello hemos seleccionado especialmente los estudios que nos proporcionarían un máximo nivel de evidencia (metaanálisis y ensayos clínicos randomizados).

Utilizaremos la palabra "niño" sin implicación genérica.

\section{TRATAMIENTO MÉDICO CONSERVADOR}

El manejo médico incluye básicamente la administración de profilaxis antibiótica (continua ó intermitente) y medidas higiénico-dietéticas.

Con excepción de los recién nacidos, en quienes la infección puede llegar al riñón por vía hematógena, en la mayoría de los casos la vía es la ascendente a partir de la flora fecal. Por este motivo todas las publicaciones sobre el tratamiento médico insisten en la importancia de la educación del niño y de su familia respecto a la higiene "perimiccional": adecuada hidratación y micciones regulares, frecuentes y completas.

El estreñimiento se ha demostrado que aumenta la virulencia de las bacterias fecales y además se considera el principal factor favorecedor de recurrencia de ITU(5), por todo ello la regularización del hábito intestinal se aconseja como medida general importante en todos los niños con RVU. Otras medidas no convencionales de profilaxis de ITU, tales como probióticos o zumo de arándanos podría jugar un papel en el manejo del RVU $(6,7)$.

El tratamiento farmacológico de la vejiga hiperactiva también forma parte del manejo médico de niños con RVU, si bien este punto no será analizado en este artículo.

El tratamiento médico está basado en el principio de que el reflujo se resuelve o disminuye de severidad espontáneamente con el tiempo en la mayoría de los niños y que las complicaciones del RVU pueden ser minimizadas con profilaxis antibiótica (8).

No hay evidencia de que el reflujo estéril dañe el riñón a no ser que la infección esté presente (con la excepción de niños con altas presiones intravesicales de forma sostenida). Por tanto, el RVU sólo, sin obstrucción ni infección, no parece ser peligroso para el parénquima renal $(9,10)$. Todo ello lleva a la creencia de que todos los niños con RVU deben de ser mantenidos en profilaxis antibiótica (1 1-13).

\section{PROFILAXIS ANTIBIÓTICA}

Las estrategias del enfoque terapéutico del RVU se fundamentan en una serie de hipótesis, no bien contrastadas, pero con una elevada aceptación: $(14-16,17)$. 
1) El reflujo predispone al niño a sufrir infecciones urinarias $y$ al desarrollo de pielonefritis.

2) El daño renal resulta de repetidos episodios de pielonefritis aguda que llevan a una potencial hipertensión arterial e insuficiencia renal.

3) La profilaxis antibiótica previene la infección hasta que el RVU es corregido quirúrgicamente ó se resuelve espontáneamente.

4) La administración de antibióticos sólo en casos de ITU no es adecuado para prevenir el daño renal.

A pesar de todo en la actualidad no existen recomendaciones universales sobre la duración de la profilaxis antibiótica $y$, aunque su prescripción es sistemática en los niños, no existe consenso sobre los medicamentos, las dosis ni las modalidades de control. Así en las indicaciones de la profilaxis antibiótica de la Asociación Americana de Urología (12) y de la Sociedad Sueca de Medicina (18) no se especifica tipo de fármaco, dosis ni intervalo.

La práctica habitual de someter a todos los niños con RVU a profilaxis antibiótica se remonta muchos años atrás cuando se comunicó la existencia de una correlación entre RVU y lesiones renales (19) y que la quimioprofilaxis reducía el riesgo de reinfecciones de orina en niños con $\operatorname{RVU}(20)$

La profilaxis antibiótica consiste en la administración continua ó discontinua de dosis reducidas de antibiótico con la finalidad de conseguir niveles urinarios suficientes para evitar la multiplicación bacteriana y el consiguiente ascenso de microorganismos hacia el tracto urinario superior. Habitualmente se administra en una única dosis nocturna si bien en niños incontinentes puede repartirse en dos tomas.

El antibiótico seleccionado para la profilaxis antibiótica debería de cumplir una serie de condiciones (21): efectividad frente a la mayoría de los uropatógenos, causar los mínimos efectos secundarios, buena tolerancia, no crear resistencias bacterianas y producir el mínimo impacto sobre la flora bacteriana.

Los fármacos clásicamente utilizados y recogidos en la literatura científica son, entre otros, nitrofurantoína, trimetoprim, trimetoprim-sulfametoxazol (Tablas I y II). Debido a las restricciones de estas sustancias en la primera infancia en muchos países y a que la nitrofurantoína y el trimetoprim-sulfametoxazol está contraindicado en el primer mes de vida, las cefalosporinas orales son preferidas en este grupo de edad. Sin embargo no está bien documentada la utilización de cefalosporinas como profilaxis de ITU.

Consideramos indicado resaltar que la mayoría de los estudios utilizan profilaxis antibiótica continua y que pocos trabajos hacen constar efectos adversos a los fármacos cuyo porcentaje se sitúa entre un $8-10 \%$, generalmente no graves (22). De todas formas se constata una creciente resistencia a los antibióticos $(23,24)$ : Un estudio encontró que a los niños a los que se le había administrado recientemente trimetoprim-sulfametoxazol durante 4 semanas ó más eran más propensos a presentar aislamientos resistentes de $E$. Coli que aquellos a los que no se les había administrado antibióticos (OR 23,4 IC95\% 12 a 47,6) (25).

\section{Prevención de las infecciones urinarias recurrentes}

Existen algunos estudios que abordan la estrategia del tratamiento antibiótico para reducir las infecciones urinarias de repetición en niños. Aunque la mayoría no se ha realizado en niños con RVU conocido, parece lógico que puedan extrapolarse parte de sus resultados ya que el reflujo formaría parte del correlato nosológico de muchos de estos niños.

Así en una revisión sistemática de la Cochrane, (26) -que valora el uso de antibióticos para la prevención de infecciones urinarias recurrentes en niños- se incluye un ensayo clínico aleatorizado (ECA) que comparaba la nitrofurantoina versus trimetoprim (incluye niños con y sin $\mathrm{RVU}$ ). La nitrofurantoína comparada con trimetoprim redujo la recurrencia de ITU a los 6 meses (RR 0,48 IC95\% 0,25 a 0,92; NNT 5, IC95\% 3 a 33). Un número mayor de niños interrumpía el tratamiento con nitrofurantoína versus trimetoprim debido a los efectos adversos que incluían naúseas, vómitos ó dolor de estómago (RR 3,17 IC95\% 1,36 a 7,37 ; NND 5, IC95\% 3 a 13). Esto indica que los efectos secundarios de la nitrofurantoína podrían superar su efecto profiláctico comparado con el trimetoprim. A destacar importantes errores metodológicos que hacen que las conclusiones se tengan que tomar con mucha precaución.

Otro estudio realizado en niños con ITU recurrentes sin reflujo, comparó cefprozil y cotrimoxazol con cefradoxilo. Este último mostró una eficacia levemente superior en el control de la infección, pero sin alcanzar diferencias estadísticamente significativas (27).

En un estudio se comparó pivmecillinam con nitrofurantoína, en una serie de niños con reflujo o infección urinaria recurrente, no evidenciándose diferencias en la tasa de recurrencias, aunque se encontraron cambios en los agentes etiológicos, con preponderancia del enterococo en los niños tratados con pivmecillinam (28). Otro ensayo clínico que comparó la eficacia del cotrimoxazol frente a la nitrofurantoína, no encontró diferencias en la prevención de las infecciones (29).

Hasta el año 2000 no se han publicado revisiones sistemáticas sobre este tema $(5,26,30)$.

En la revisión de Le Saux (5) se incluyen 6 ECA ( 3 con niños con vejiga neurógena y 3 con niños con tracto urinario normal) que comparan cualquier tipo de antibiótico para la profilaxis antibiótica (administrado por un período de 3 meses ó más) con placebo ó no antibióticos y se evalúa el número de ITU.

La calidad de los ECA es deficiente. Uno de los $E C A$, que incluye niños con vejiga neurógena, no ofreció información útil.

Los principales resultados destacan la menor incidencia de infecciones en el grupo con profilaxis en pa- 
cientes con tracto urinario normal, que era 0 a 4 por cada 10 pacientes y año comparado con las de 4 a 16,7 en el grupo control. La tasa de recurrencia en pacientes con vejiga neurógena (en dos ensayos) fue de 2,9 a 17,1 por cada 10 pacientes y año en el grupo de tratamiento y de 1,5 a 33 en el grupo control. Los autores comentan que es preocupante la sorprendente falta de información en niños con RVU.

Otra revisión sistemática (26) evalúa el uso prolongado de antibióticos para la prevención de infecciones recurrentes en niños. Incluye tres ECA (151 niños de menos de 18 años con riesgo de infección del tracto urinario) que comparan los antibióticos (nitrofurantoína, trimetoprim - sulfametoxazol) frente a ningún tratamiento ó placebo para prevenir la recurrencia de infección urinaria en niños. A destacar que se trata de una población que incluye pacientes con y sin RVU. Los ECA (publicados entre los años 1975-1978) variaban en cuanto a la duración de la profilaxis antibiótica (10 semanas a 12 meses) y en el método de encubrimiento de la asignación a diferentes intervenciones. Aquí también encuentran que en comparación con el placebo ó ningún tratamiento, los antibióticos disminuyeron el riesgo de infección urinaria recurrente (RR $0,36[0,16-0,77])$.

No se describieron efectos secundarios en ninguno de estos tres ensayos.

Se trata de una revisión sistemática exhaustiva pero que identificó ECA metodológicamente débiles. Ninguno de los ECA incluidos en la revisión sistemática utilizó análisis por intención de tratar. Solamente uno tenía el encubrimiento adecuado de la asignación a las diferentes intervenciones y sólo uno especificó las medidas de resultado. Por lo tanto las conclusiones que de ella se obtengan son cuestionables.

\section{Profilaxis en niños con RVU}

Parecía demostrado cierto efecto protector de los antibióticos tomados de forma continuada, para disminuir las infecciones urinarias recurrentes. A partir de ahí la Asociación Americana de Urología publicó en 1997 recomendaciones sobre el tratamiento del RVU infantil. Este trabajo cita varios metaanálisis y aconseja la profilaxis antibiótica como el tratamiento inicial para todos los niños hasta los 5 años de edad con RVU de grado I a IV diagnosticados después de una infección urinaria (12). Estas indicaciones no coinciden con la Sociedad Sueca de Medicina que sólo la indica para aquellos niños con RVU de grado III, IV y V en el examen inicial ya que para los grados I y I| recomiendan medidas higiénicas (18).

Estas recomendaciones terapeúticas que se han mantenido durante muchos años eran fruto de la extrapolación de estudios no realizados en niños con RVU y basados prioritariamente en opinión de expertos.

Es importante resaltar que la mayoría de los estudios que se han publicado en niños con RVU han comparado la profilaxis antibiótica con la combinación de profilaxis antibiótica y cirugía ó la profilaxis antibiótica con la cirugía aislada y no el tratamiento médico aislado (31-36).

La calidad de la mayoría de los estudios realizados sobre el uso de la profilaxis antibiótica en niños con RVU no está bien contrastada. La mayoría incluyen defectos metodológicos o excluyen la comparación con placebo por razones éticas. Esto ha provocado que carezcamos de estudios sólidos para llegar a conclusiones válidas. De todas formas contamos con dos ensayos clínicos que presentan resultados en un mismo sentido.

Un estudio publicado como abstract en Proceedings de American Academy of Pediatrics en 1997 (37) que valora intervenciones para el RVU primario, compara en tres ramas, dos modalidades de administración antibiótica (profilaxis diaria o profilaxis administrada tres días por semana) frente a la vigilancia clínica con tira reactiva de orina de control diaria, sobre un total de 43 niños con RVU (no se especifica el grado). No existieron diferencias significativas en el riesgo de infección urinaria entre la profilaxis antibiótica diaria y ninguna profilaxis (RR 0,25 $[0,03-1,83])$ o entre la profilaxis intermitente y ninguna profilaxis (RR 0,46 [0,10-2]).

Tampoco existieron diferencias significativas en el riesgo de lesión del parénquima renal entre la profilaxis antibiótica diaria y ninguna profilaxis ( $\operatorname{RR~} 0,40[0,02-9,18]$ ) o entre la profilaxis intermitente y ninguna profilaxis (RR $0,38[0,02-8,59])$.

No se describieron resultados sobre efectos secundarios.

Un nuevo estudio mas reciente publicado por Garin y colaboradores (38) analiza en un ensayo clínico bien diseñado el efecto de la profilaxis sobre la infección.

Se trata de un ensayo clínico aleatorizado, controlado y multicéntrico cuyo objetivo es evaluar el papel del RVU y de la profilaxis antibiótica en las recurrencias de ITU y daño parenquimatoso renal después de un episodio de pielonefritis aguda (PNA).

Incluye 236 niños (entre 3 meses y 18 años de edad) con PNA con ó sin RVU leve-moderado (grados I,II y III). Se analizaron los 218 pacientes que cumplieron 1 año de seguimiento. De ellos consideramos el subgrupo de los 113 niños con RVU (55 niños con profilaxis y 58 sin profilaxis).

Estudia la comparación del efecto entre la profilaxis antibiótica continua frente a la vigilancia clínica y tratamiento precoz de la infecciones agudas, valorando el número de infecciones urinarias y el daño parenquimatoso renal. No se utilizó placebo.Los principales resultados aparecen resumidos en la Tabla III.

No hay diferencias en el número total de infecciones recurrentes entre ambos grupos de pacientes, tras un año seguimiento. Un $23,6 \%$ de 55 niños con profilaxis antibiótica presentó alguna infección urinaria, frente a un $22,4 \%$ de 58 niños sin tratamiento de mantenimiento (RR $1,05[0,54-2,07])$. 
Los niños con reflujo acompañados de profilaxis antibiótica presentan mas episodios de pielonefritis en seguimiento a un año ( 7 casos en 55 niños) que los niños sin profilaxis antibiótica (1 caso en 58 niños), pero sin alcanzar significación estadística (RR 7,38 [0,93-58,06]).
Tampoco hay diferencias en el riesgo de aparición de lesiones parenquimatosas renales tras la primera pielonefritis entre ambos grupos de pacientes. En un $9 \%$ de 55 niños con profilaxis antibiótica apareció alguna lesión parenquimatosa renal (identificada por gammagrafía

TABLA I. MODALIDADES DE PROFILAXIS EN LOS PRINCIPALES ESTUDIOS.

\begin{tabular}{|c|c|c|c|c|}
\hline & Tipo de antibiótico y dosis & $\begin{array}{c}\text { Continua } \\
o ́ \\
\text { discontinua }\end{array}$ & Duración & $\begin{array}{c}\text { Efectos } \\
\text { adversos }\end{array}$ \\
\hline $\begin{array}{c}\text { Asociación } \\
\text { Americana } \\
\text { de Urología (12) }\end{array}$ & & Continua & & \\
\hline $\begin{array}{c}\text { Sociedad } \\
\text { Sueca } \\
\text { de } \\
\text { Medicina (18) }\end{array}$ & & & $\begin{array}{l}\text { - RVU grado V todas las } \\
\text { edades y grado IV bilateral } \\
\text { en < } 1 \text { año en estudio ini- } \\
\text { cial: Al menos } 1 \text { año y luego } \\
\text { en función de exploraciones. } \\
\text { - RVU grado III y IV en estu- } \\
\text { dio inicial: al menos } 1 \text { año. } \\
\text { - RVU grado III y IV en el } \\
\text { seguimiento: } \\
\text { - Niñas: profilaxis (no } \\
\text { específica) ó cirugía. } \\
\text { - Niños: stop profilaxis }\end{array}$ & \\
\hline $\begin{array}{l}\text { Le Saux (5) } \\
\text { Antibiótico vs } \\
\text { placebo ó no } \\
\text { antibiótico. } \\
\text { (3 estudios sin } \\
\text { vejiga } \\
\text { neurógena) }\end{array}$ & $\begin{array}{l}\text { - Diseño Cruzado } \\
\text { Profilaxis: } \\
\text { NFN: 1,2-2,4mg/kg/día } \\
\text { Control: Placebo } \\
6 \text { meses en cada período } \\
\text { - ECAC } \\
\text { Profilaxis: } \\
\quad \text { Cotrimoxazol (2mgTMP/ } \\
\quad 10 \text { mg SMX por kg/día; } \\
\text { 6-12 meses ó NFN: 1-2 } \\
\text { mg/kg/día; 6-12 meses. } \\
\text { Control: No profilaxis } \\
\text { - Diseño Cruzado } \\
\text { Profilaxis: NFN } \\
\quad 1 \text { mg/kg/día } \\
\text { Control: OM-89; 6mg/día } \\
6 \text { meses en cada período }\end{array}$ & 更 & $\begin{array}{c}\text { Oscila entre } \\
3 \text { meses-12 meses }\end{array}$ & $\begin{array}{c}\text { No efectos } \\
\text { adversos }\end{array}$ \\
\hline
\end{tabular}

RVU: Reflujo vesicoureteral; NFN: Nitrofurantoína; SMX: sulfametoxazol; TMP:Trimetoprim

OM-89: extracto proteínico liofilizado de bacterias (E. Coli); ECAC: ensayo clínico aleatorizado controlado. 
renal-DMSA-), en comparación con el 3,4\% de 58 niños sin profilaxis antibiótica (RR 2,64 [0,53-13,03]).

La tasa de resolución del RVU (leve a moderado) al cabo de un año de seguimiento no difirió significativamente entre los grupos con o sin profilaxis antibiótica.
Por tanto sus resultados no apoyan el empleo de la profilaxis antibiótica para prevenir la recurrencia de la infección ni el desarrollo de cicatrices renales. Incluso la profilaxis aumentó la probabilidad de desarrollar una pielonefritis.

TABLA II. MODALIDADES DE PROFILAXIS EN LOS PRINCIPALES ESTUDIOS.

\begin{tabular}{|c|c|c|c|c|}
\hline & Tipo de antibiótico y dosis & $\begin{array}{c}\text { Continua } \\
o ́ \\
\text { discontinua }\end{array}$ & Duración & Efectos adversos \\
\hline $\begin{array}{c}\text { Williams (26) } \\
\text { Antibiótico vs } \\
\text { ningún tratamiento } \\
\text { ó placebo } \\
\text { (3 ECA) }\end{array}$ & $\begin{array}{l}\text { - Profilaxis: } \\
\text { NFN: 4mg/kg/día, } \\
10 \text { semanas ó TMP-SMX } \\
\text { 20- } 40 \text { mg TMP/ } \\
\text { 100-200mg SMX, } \\
2 \text { veces/día, } 10 \text { semanas } \\
\text { (después de } 2 \text { semanas de } \\
\text { tratamiento agudo). } \\
\text { Control: No profilaxis (después de } \\
2 \text { semanas de tratamiento agudo } \\
\text { con ampicilina) } \\
\text { - Profilaxis } \\
\text { Cotrimoxazol:2 mg TMP/10 mg } \\
\text { SMX kg/día; } 6-12 \text { meses } \\
\text { ó NFN: } 1-2 \text { mg/kg/día;6-12 meses } \\
\text { Control: No profilaxis } \\
\text { - Profilaxis:Cotrimoxazol (no se } \\
\text { especifica dosis); } 6 \text { meses. } \\
\text { Control: Placebo } 6 \text { meses }\end{array}$ & Continua & $\begin{array}{c}10 \text { semanas } \\
a \\
12 \text { meses }\end{array}$ & $\begin{array}{l}\text { No se describieron efec- } \\
\text { tos adversos en ninguno } \\
\text { de los } 3 \text { ensayos }\end{array}$ \\
\hline $\begin{array}{c}\text { Williams (26) } \\
\text { Nitrofurantoína vs } \\
\text { trimetoprim } \\
\text { (1 ensayo doble } \\
\text { ciego) }\end{array}$ & $\begin{array}{l}\text { - NFN: } 1-1,5 \mathrm{mg} / \mathrm{kg} ; 6 \text { meses. } \\
\text { - TMP:2-3 mg } / \mathrm{kg} ; 6 \text { meses. }\end{array}$ & Continua & 6 meses & $\begin{array}{l}\text { Nitrofurantoina: efectos } \\
\text { secundarios, sobre todo } \\
\text { del aparato digestivo. }\end{array}$ \\
\hline Reddy (37) & No se especifica & $\begin{array}{l}\text { Continua vs } 3 \\
\text { veces/sem vs } \\
\text { no profilaxis }\end{array}$ & 1 año & $\begin{array}{c}\text { No se describieron } \\
\text { resultados sobre efectos } \\
\text { adversos }\end{array}$ \\
\hline Garin (38) & $\begin{array}{l}\text { Profilaxis:TMP-SMX:1-2mg TMP } \\
\text { y } 5-10 \mathrm{mg} \text { SMX/kg; } 1 \text { vez/día ó } \\
\text { NFN: } 1,5 \mathrm{mg} / \mathrm{kg} ; 1 \text { vez/ día. } \\
\text { Control: No profilaxis }\end{array}$ & $\begin{array}{c}\text { Continua } \\
\text { vs } \\
\text { no profilaxis }\end{array}$ & 1 año & No efectos adversos \\
\hline
\end{tabular}

ECA:Ensayo clínico aleatorizado; NFN:Nitrofurantoína; TMP:Trimetoprim. SMX:Sulfametoxazol. 
TABLA III. RECURRENCIA DE ITU Y PRESENCIA DE CICATRICES RENALES EN EL SUBGRUPO DE NIÑOS CON RVU 11 13) EN EL ESTUDIO DE GARIN Y COLS. (38).

\begin{tabular}{|l|c|c|}
\hline & Con profilaxis (55) & Sin profilaxis (58) \\
\hline Recurrencia de ITU & 0 & 3 \\
\hline Asintomático & 6 & 9 \\
\hline Cistitis & 7 & 1 \\
\hline Pielonefritis aguda & 42 & 45 \\
\hline Ninguna & & 2 \\
\hline Cicatrices renales & 5 & 56 \\
\hline SI & 50 & \\
\hline NO & & \\
\hline
\end{tabular}

ITU: Infección del tracto urinario; RVU: Reflujo vesicoureteral.

\section{Reflexiones finales}

Llama la atención la escasa evidencia científica disponible que valore la eficacia de un tratamiento tan aceptado universalmente desde hace tanto tiempo.

El que no esté demostrada suficientemente la eficacia de la profilaxis antibiótica para prevenir las infecciones de orina y el daño renal no quiere decir que no pueda ser beneficiosa en determinadas situaciones. Se requiere la realización de estudios bien diseñados y rigurosos para poder dar unas recomendaciones. De hecho con la idea de conseguir un manejo óptimo del RVU se deduce claramente que es necesario que se pongan en marcha más ensayos como los de Garin y cols. (38); además se necesitan estudios que evaluen qué ocurre con RVU de alto grado.

Sería deseable que se haga un estudio prospectivo comparando profilaxis antibiótica y entrenamiento miccional con entrenamiento miccional sin profilaxis para determinar la incidencia de infecciones urinarias altas $y$ bajas y si hay alguna diferencia en el daño renal porque algunos niños podrían no necesitar ni cirugía ni profilaxis.

Sin duda, algunos niños se beneficiarán de un tratamiento quirúrgico cuyas posibles indicaciones se escapan al ámbito de este artículo.

Consideramos importante enfatizar que no hay datos disponibles que demuestren la superioridad del trata- miento médico ó quirúrgico comparado con la observación y tratamiento precoz de las infecciones de orina, que sería también otra opción de tratamiento en el manejo del RVU primario en el niño.

\section{BIBLIOGRAFÍA y LECTURAS RECOMENDADAS (*lectura de interés $y^{* *}$ lectura fundamental)}

1. RANSLEY, P.G.; RISDON, R.A.: "Reflux and renal scarring". Br. J. Radiol. Suppl., 14: 1, 1978.

2. JAKOBSSON, B.; NOLSTEDT, L.; SVENSSON, L. y cols.: "99mTechnetium-dimercaptosuccinic acid scan in the diagnosis of acute pyelonephritis in children: Relation to clinical and radiological findings". Pediatr. Nephrol., 6: 328, 1992.

3. RUSHTON, H.G.; MAJD, M.; JANTAUSCH, B. у cols.: "Renal scarring following reflux and nonreflux pyelonephritis in children: Evaluation with 99mtechnetium-dimercaptosuccinic acid scintigraphy". J. Urol., 147: 1327, 1992.

4. HOBERMAN, A.; CHARRON, M.; HICKEY, R.W. y cols.: "Imaging studies after a first febrile urinary tract infection in young children". N. Engl. J. Med., 348: 195, 2003.

**5. LE SAUX, N.; PHAM, B.; MOHER, D.: "Evaluating the benefits of antimicrobial prophylaxis to prevent uri- 
nary tract infections in children: A systematic review". CMAJ, 163: 523, 2000.

6. KERR, K.G.: "Cranberry juice and prevention of recurrent urinary tract infection". Lancet., 353: 673, 1999.

7. KONTIOKARI, T.; SUNDQVIST, K.; NUUTINEN, M. y cols.: "Randomised trial of cranberry-lingonberry juice and Lactobacillus GG drink for the prevention of urinary tract infections in women". BMJ, 322: 1571, 2001.

8. ELDER, J.S.: "Guidelines for consideration for surgical repair of vesicoureteral reflux". Curr. Opin. Urol., 10: 579, 2000.

9. RANSLEY, P.G.; RISDON, R.A.: "The pathogenesis of reflux nephropathy". Contrib. Nephrol., 16: 90, 1979.

10. BELMAN, A.B.: "A perspective on vesicoureteral reflux”. Urol. Clin. North Am., 22: 139, 1995.

11. ELDER, J.S.; SNYDER, H.M.; PETERS, C. y cols.: "Variations in practice among urologists and nephrologists treating children with vesicoureteral reflux". J. Urol., 148: 714, 1992.

**12. ELDER, J.S.; PETERS, C.A.; ARANT, B.S. Jr. y cols.: "Pediatric Vesicoureteral Reflux Guidelines Panel summary report on the management of primary vesicoureteral reflux in children”. J. Urol., 157: 1846, 1997.

13. JODAL, U.; KOSKIMIES, O.; HANSON, E. y cols.: "Infection pattern in children with vesicoureteral reflux randomly allocated to operation or long-term antibacterial prophylaxis. The International Reflux Study in Children". J. Urol., 148: 1650, 1992.

**14. GARIN, E.H.; CAMPOS, A.; HOMSY, Y.: "Primary vesicoureteral reflux: Review of current concepts". Pediatr. Nephrol., 12: 249, 1998.

**15. FERNÁNDEZ, J.M.; MÁLAGA, S.: “Es posible cambiar el paradigma reflujocéntrico?”. Evid. Pediatr., 2: 17, 2006.

16. BUNDY, D.G.: "Vesicoureteral reflux". Pediatr. Rev., 28: 6, 2007.

*17. DEMEDE, D.; CHEIKHELARD, A.; HOCH, M. y cols.: "Evidence-based medicine and vesicoureteral reflux". Ann. Urol. (Paris), 40: 161, 2006.

**18. JODAL, U.; LINDBERG, U.: "Guidelines for management of children with urinary tract infection and vesico-ureteric reflux. Recommendations from a Swedish state-of-the-art conference. Swedish Medical Research Council". Acta Paediatr. Suppl., 88: 87, 1999.

19. HODSON, C.J.; EDWARDS, D.: "Chronic pyelonephritis and vesico-ureteric reflex". Clin. Radiol., 11: 219, 1960.

20. NORMAND, I.C.; SMELLIE, J.M.: "Prolonged maintenance chemotherapy in the management of urinary infection in childhood". Br. Med. J., 1: 1023, 1965.

*21. BOLLGREN, I.: "Antibacterial prophylaxis in children with urinary tract infection". Acta Paediatr. Suppl., 88: 48, 1999.

*22. UHARI, M.; NUUTINEN, M.; TURTINEN, J.: “Adverse reactions in children during long-term antimicrobial therapy". Pediatr. Infect. Dis. J., 15: 404, 1996.

23. MURRAY, B.E.; RENSIMER, E.R.; DUPONT, H.L.: "Emergence of high-level trimethoprim resistance in fecal Escherichia coli during oral administration of trimethoprim or trimethoprim-sulfamethoxazole". N. Engl. J. Med., 306: 130, 1982.

24. ABDEL-HAQ, N.; ABUHAMMOUR, W.; ASMAR,
B. y cols.: "Nasopharyngeal colonization with Streptococcus pneumoniae in children receiving trimethoprimsulfamethoxazole prophylaxis". Pediatr. Infect. Dis. J., 18: 647, 1999.

25. ALLEN ,U.D.; MAcDONALD, N.; FUITE, L. y cols.: "Risk factors for resistance to "first-line" antimicrobials among urinary tract isolates of Escherichia coli in children”. CMAJ, 160: 1436, 1999.

**26. WILLIAMS, G.J.; WEI, L.; LEE, A. y cols.: "Uso prolongado de antibióticos para la prevención de infecciones urinarias recurrentes en niños". La Biblioteca Cochrane Plus, 2: 1534, 2006.

27. BELET, N.; ISLEK, I.; BELET, U. y cols.: "Comparison of trimethoprim-sulfamethoxazole, cephadroxil and cefprozil as prophylaxis for recurrent urinary tract infections in children". J. Chemother., 16: 77, 2004.

28. CARLSEN, N.L.; HESSELBJERG, U.; GLENTING, P.: "Comparison of long-term, low-dose pivmecillinam and nitrofurantoin in the control of recurrent urinary tract infection in children. An open, randomized, cross-over study". J. Antimicrob. Chemother., 16: $509,1985$.

29. HOLLAND, N.H.; KAZEE, M.; DUFF, D. y cols.: "Antimicrobial prophylaxis in children with urinary tract infection and vesicoureteral reflux". Rev. Infect., 4: 467, 1982.

**30. WILLIAMS, G.; LEE, A.; CRAIG, J.: "Antibiotics for the prevention of urinary tract infection in children: A systematic review of randomized controlled trials". J. Pediatr., 138: 868, 2001.

31. SMELLIE, J.M.; TAMMINEN-MOBIUS, T.; OLBING, H. y cols.: "Five-year study of medical or surgical treatment in children with severe reflux: Radiological renal findings. The International Reflux Study in Children". Pediatr. Nephrol., 6: 223, 1992.

**32. WHEELER, D.; VIMALACHANDRA, D.; HODSON, E.M. y cols.: "Antibiotics and surgery for vesicoureteric reflux: A meta-analysis of randomised controlled trials". Arch. Dis. Child., 88: 688, 2003.

*33. DE LA PEÑA ZARZUELO, E.: "Primary vesicoureteral reflux treatment in childhood: Comparsion of two systematic review". Actas Urol. Esp., 29: 138, 2005.

34. JODAL, U.; SMELLIE, J.M.; LAX, H. y cols.: "Tenyear results of randomized treatment of children with severe vesicoureteral reflux. Final report of the International Reflux Study in Children”. Pediatr. Nephrol., 21: 785, 2006.

**35. ESPINOLA, B.; VAZQUEZ MARTUL, M.: "Vesicoureteral reflux. Old and new aspects". Nefrologia, 25: 462,2005

**36. WHEELER, D.M.; VIMALACHANDRA, D.; HODSON, E.M. y cols.: "Intervenciones para el reflujo vesicoureteral primario". La Biblioteca Cochrane Plus, 3: 1532, 2005.

**37. REDDY, P.P. y cols.: "Antimicrobial prophylaxis in children with vesico-ureteral reflux: A randomized prospective study of continuous therapy vs intermittent therapy vs surveillance". Pediatrics, 100: 555, 1997.

**38. GARIN, E.H.; OLAVARRIA, F.; GARCIA NIETO, V. y cols.: "Clinical significance of primary vesicoureteral reflux and urinary antibiotic prophylaxis after acute pyelonephritis: A multicenter, randomized, controlled study”. Pediatrics, 117: 626, 2006. 